\title{
Into the eyes of bone marrow-derived mesenchymal stem cells therapy for myocardial infarction and other diseases
}

\author{
Jian-Rui Li, Ting-Ting Qu \\ Department of Orthopedics, Dongfang Hospital Affiliated to Tongji University, Shanghai 200120, China \\ Contributions: (I) Conception and design: All authors; (II) Administrative support: None; (III) Provision of study material or patients: JR Li; (IV) \\ Collection and assembly of data: JR Li; (V) Data analysis and interpretation: All authors; (VI) Manuscript writing: All authors; (VII) Final approval of \\ manuscript: All authors. \\ Correspondence to: Jian-Rui Li, MM. Department of Orthopedics, Dongfang Hospital Affiliated to Tongji University, Shanghai 200120, China. \\ Email: Doctocracy_Lee@163.com.
}

\begin{abstract}
Applications of bone marrow-derived mesenchymal stem cells (BM-MSCs) have been documented for diseases occur in the sports system, the central nervous system, the cardiovascular system etc. However, poor viability of donor stem cells after transplantation limits their therapeutic efficiency. Although the autophagy theory has been reported, the underlying mechanisms are still poorly understood. Isolation and culture methods of mesenchymal stem cells are currently concentrate on four ways. Overall, BM-MSCs have both important research significance and clinical application value in cell replacement therapy, gene therapy and reconstruction of tissues as well as organs especially for myocardial infarction (MI). In this article, we review the biological characteristics of BM-MSCs and its research progress especially in MI.
\end{abstract}

Keywords: Autophagy; bone marrow-mesenchymal stem cells (BM-MSCs); apoptosis; hypoxia; signal transduction pathways; myocardial infarction (MI)

Received: 23 February 2017; Accepted: 13 July 2017; Published: 23 August 2017.

doi: $10.21037 /$ sci.2017.08.01

View this article at: http://dx.doi.org/10.21037/sci.2017.08.01

\section{Introduction}

Bone marrow mesenchymal stem cells (BM-MSCs) are mesoderm derived stem cells, which mainly exist in the interstitial connective tissue. Bone marrow tissue has the most abundant content of BM-MSCs. Due to characteristic of self-renewal, proliferation and multi-directional differentiation in appropriate micro environment, BMMSCs have the potential to promote the repair of tissue injury. In view of the advantages of BM-MSCs which are easy to obtain, cultivate, and they have low immunogenicity, which can survival for a long-term in the host and easy for exogenous gene transfer as well as long-term expression, BM-MSCs have been widely used in the field of tissue engineering, cell transplantation, gene therapy, and organ transplantation. Studies showed that the surface antigen phenotype of mesenchymal stem cells was not single but had the characteristics of mesenchymal, endothelial and muscle cells. Besides, immunohistochemistry and flow cytometry revealed that SH2, SH3, CD71, CD29, CD44, CD90, and CD120A were all positive expressed, which can be generally used to identify and amplify the mesenchymal stem cells (1). Recent studies showed that BM-MSCs could be used in the clinical treatment of autoimmune diseases, degenerative diseases and hypoxic ischemic brain damage $(2,3)$. Bone marrow not only contains hematopoietic stem cells which can develop and differentiate into all types of blood cells but also has mesenchymal stem cells which can produce non hematopoietic tissues. Some of articles also called them stick wall cells or fibroblast colony forming units as they relatively easy to adherent and form into fibroblast like clones. Moreover, as BM-MSCs come from the supporting structure of the bone marrow, they can act as feeder layer to support growth of hematopoietic stem cells. Therefore BM-MSCs are also called bone marrow stromal cells. In view of the following features of 
bone marrow which has aroused people's interest $(1,4,5)$. Furthermore, BM-MSCs can act as support hematopoietic cells, promoting the growth of hematopoietic stem cells. Above all, BM-MSCs have broad application prospects in tissue engineering, cell transplantation and gene therapy because of the advantages of easy separation, amplification and easy operation in vitro and in vivo (6-9). Taken together, BM-MSCs have important research significance and clinical application value in cell replacement therapy, gene therapy and tissue regeneration. In this article we review the latest progress, limitation as well as clinical application of BMMSCs.

\section{Isolation of BM-MSCs}

BM-MSCs are obtained mainly from bone marrow aspiration, while human BM-MSCs are generally drawn from the anterior superior iliac spine (10). They are also available from the tibia, femur, sternum, lumbar spine. The acquisition sites of BM-MSCs in large animals are the same as humans, however, rabbits' BM-MSCs need to be extracted in the middle of the tibia or femur bone marrow (11-13). The proportion of BM-MSCs nucleated cell population accounts for less than $0.0001 \%$ of them, however they can easily be isolated and expanded by using certain cell culture techniques (10). Stro-1 monoclonal antibody is usually used to isolate BM-MSCs which grow by attaching to the wall in laminin adhesion culture plate with low concentration of serum and CD45-/A-glycoproteins (14). In the past, BM-MSCs isolation methods were mainly concentrated on density gradient centrifugation method, differential adherence screening method, flow cytometry sorting method as well as immune beads method (15). However, high purity of BM-MSCs cannot be obtained by the four kinds of separation methods as described above. Nowadays, selecting the appropriate factor based on different reactivity of BM-MSCs to growth factors, to stimulate the proliferation, obtaining a higher proportion the mesenchymal stem cells has been regarded as a more accurate isolation method. Meanwhile, the new method of using 3 microns diameter plastic petri dish to screen BMMSCs, whose homogeneity are greater than $98 \%$, with capacity of proliferation, self-renewal and have the potential to differentiate into bone, fat, cartilage tissue differentiation nature (6,16-20). Overall, currently methods are various among laboratories in the world and further standardization of the BM-MSCs' separation process is still needed indepth study according to their biological characteristics and mechanisms.

\section{Application of BM-MSCs: update}

Based on its far-reaching biological effects, there are increasingly number of researches and exciting discoveries since 1999 (21) (Figure 1). Up to now, applications of BMMSCs towards cells, animals and clinical tests have been came down to diseases occurred in the sports system (21), the central nervous system (4), the cardiovascular system (21), the respiratory system (21), the digestive system (22), the urinary system (21) etc. (Figure 2). Among these systems, researches about the sports system and the central nerves system account for a significant proportion. It's interesting to note that there is one paper tried to treat cancer by using BM-MSCs, which has the great significance as most therapies toward cancers have various kinds of severe complications in patients (23).

\section{Autophagy and apoptosis in the BM-MSCs therapy towards myocardial infarction (MI)}

Autophagy is a process of intracellular bulk degradation in which cytoplasmic components including organelles are sequestered within double-membrane vesicles that deliver the contents to the lysosome/vacuole for degradation $(24,25)$. There are three primary forms of autophagy: chaperonemediated autophagy, microautophagy and macroautophagy (24-28). During the process of macroautophagy, the sequestering vesicles, termed autophagosomes, fuse with the lysosome or vacuole resulting in the delivery of an inner vesicle (autophagic body) into the lumen of the degradative compartment (29-31).

Nowadays, one of the highest incidence and fatality rate of clinical disease is MI (32-35). Numerous studies have been proved BM-MSC is an emerging effective therapy to the disease. However, poor viability of donor stem cells after transplantation limits their therapeutic efficiency, whereas, the underlying mechanism is still poorly understood (36). Autophagy, a highly conserved process of cellular degradation, is required for maintaining homeostasis and normal function including MI (37).

Actually, when it comes to the role of autophagy on cell, it is still a controversial issue according to the present study $(11,38-41)$. There is evidence demonstrated that autophagy can either protect cells or contribute to cell death depend on the intensity of stimulus. Autophagy at basal levels is involved in maintaining normal function in various 


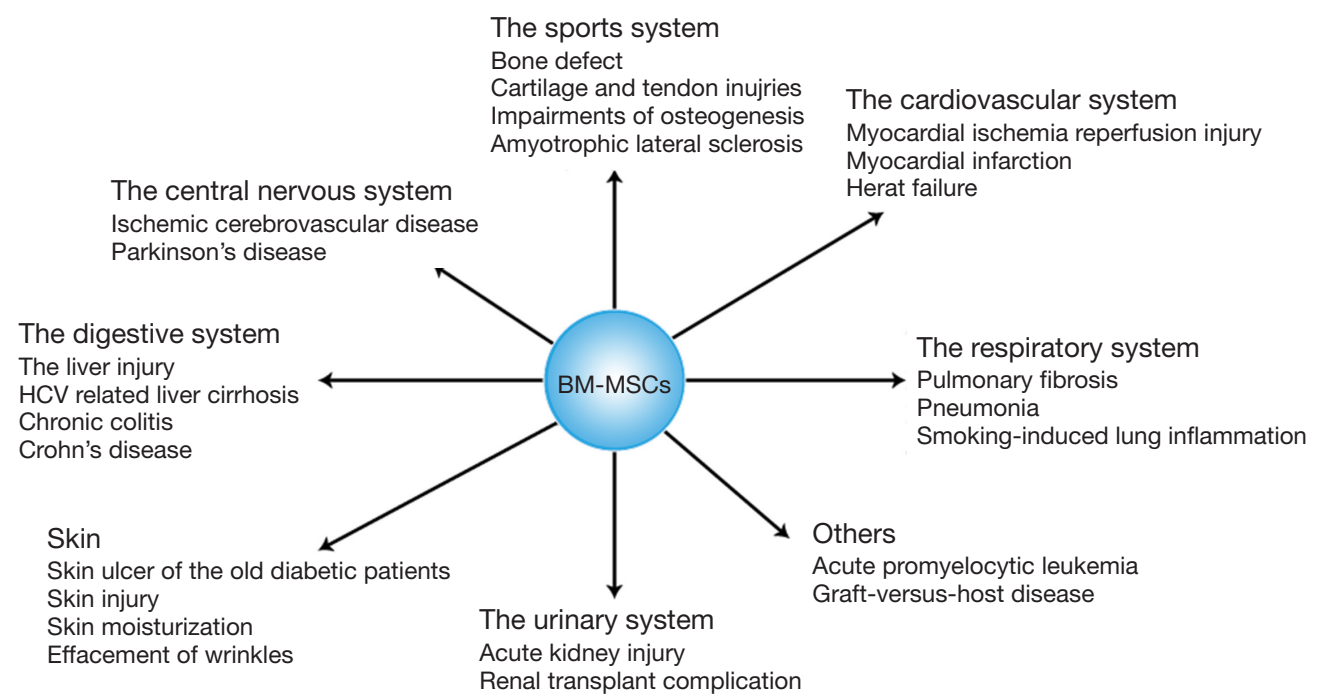

Figure 1 Summary of potential of various preventive and therapeutic effects of BM-MSCs. BM-MSCs, bone marrow-mesenchymal stem cells.

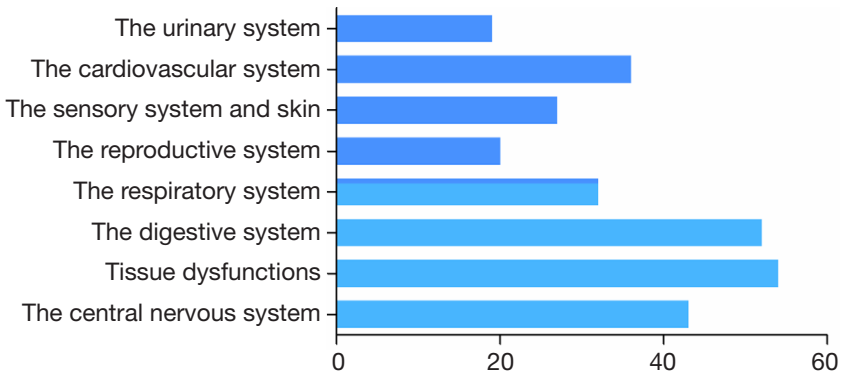

Figure 2 Number of papers that report effects of BM-MSCs in various systems between 2012 and 2016. BM-MSCs, bone marrowmesenchymal stem cells.

organisms. Hence, autophagy has been generally considered as a protective cellular response against various stresses. Previous studies have demonstrated that modest autophagy induced by sublethal hypoxic preconditioning can increase cell survival and inhibit extensive apoptosis $(41,42)$.

Conversely, other studies also suggested that extensive and prolonged autophagy may be a promoter of apoptosis, leading to cell death as type II programmed cell death. Such discrepancy may be attributed to differences in hypoxic treatment protocol. Our previous studies adopted hypoxia $\left(1 \% \mathrm{O}_{2}\right)$ /serum deprivation injury for $24 \mathrm{~h}$ to mimic ischemic microenvironment in vivo (43). However, Liu et al. performed hypoxic preconditioning with $5 \% \mathrm{O}_{2}$ for $6 \mathrm{~h}$ (44). The comparison results showed that autophagy is paradoxical that can both protect and impair cell survival depending on the environment. Therefore, the different stress or anaerobic injury may result in the disparate effects of autophagy on MSCs.

Regulating autophagic activity may be a potential optimizing target for promoting BM-MSCs based cellular therapy for MI (43). Thus, many signaling pathways have been suggested to participate in autophagy regulation. As the main problem of stem cell therapy is that the survival ability of implanted stem cells is poor, the survival mechanism and related regulation are more and more concerned by people. Until now, there are some potential signal transduction pathways such as PI3K/Akt/nuclear factor-kappa B, MEK/ERK and SCF/c-kit which participate in the relationship between autophagy and apoptosis has been reported to participate in autophagy. Besides, autophagy plays a key role in promoting the survival of transplanted stem cells in MI and it may provide a new 
therapeutic approach for stem cell therapy and regenerative medicine (45).

\section{Remaining problems and future directions}

In recent years, studies of BM-MSCs have been made great progresses, but there are still some problems to be solved. Firstly, no standard method has been putted forward about isolation, purification and specific marker molecules for the identification of BM-MSCs. Secondly, the efficiency of BM-MSCs differentiation is not ideal, which is currently one of the research focus on how to induce BM-MSCs to differentiate to the single specific tissue and cells (46-49). Thirdly, the signal transduction mechanism and the molecular basis of BM-MSCs' differentiation such as which transcription factors or gene were activated to make it for some specific differentiation is still not clear though it is generally considered to be related to reprogramming of BM-MSCs. Fourthly, whether the induced cells have real structure and function, homing to the corresponding organization are need to be further investigated. Lastly, although there is no report on the transformation of BMMSCs into malignant cells and the production of abnormal extracellular matrix, the safety of BM-MSCs is also worth noting.

On the other hand, BM-MSCs have important clinical application value in cell replacement therapy, gene therapy and tissue regeneration. Bone marrow collection is also convenient, safe and cause less injury, especially has no obvious complications to the donor. Therefore BMMSCs is conducive to the expansion and autologous transplantation in vitro, thereby becoming the promising source of tissue engineering. BM-MSCs were amplified in vitro, which can directly carry on cell transplantation or implant biomaterials, and then transplanted into the body to repair tissue defects. In addition, retroviral vector, adenovirus vector which carry the target gene can be also successfully transfected into BM-MSCs, and have high expression in vivo. Besides, BM-MSCs are relatively primitive cells, whose immunogenicity is weak and can inhibit the mixed lymphocyte reaction. However, previous studies also reported the clinical application of BM-MSCs is not matched with donor, host immune rejection or graft versus host reaction (50-52). Moreover, allogeneic bone marrow transplantation of mesenchymal stem cells in severe idiopathic aplastic anemia patients have also been shown to improve the effect of bone marrow stromal function $(53,54)$. Allogeneic transplantation of BM-MSCs not only has ability of multi-directional differentiation, but also has special immune tolerance, allowing them to survive in allogeneic environment, in which provides a possibility for the application of BM-MSCs MI therapy.

\section{Acknowledgements}

The authors would like to thank the authorities of Dongfang Hospital Affiliated to Tongji University, Shanghai, China for providing necessary facilities to conduct the review article.

\section{Footnote}

Conflicts of Interest: The authors have no conflicts of interest to declare.

\section{References}

1. Pittenger MF, Martin BJ. Mesenchymal stem cells and their potential as cardiac therapeutics. Circ Res 2004;95:9-20.

2. Liu $Y$, Zhang X, Dai Y, et al. [Effects of bone marrow mesenchymal stem cells on learning and memory functional recovery in neonatal rats with hypoxic-ischemic brain damage]. Zhonghua Er Ke Za Zhi 2008;46:648-53.

3. Togha M, Jahanshahi M, Alizadeh L, et al. Rapamycin Augments Immunomodulatory Properties of Bone Marrow-Derived Mesenchymal Stem Cells in Experimental Autoimmune Encephalomyelitis. Mol Neurobiol 2017;54:2445-57.

4. Nakano M, Nagaishi K, Konari N, et al. Bone marrowderived mesenchymal stem cells improve diabetes-induced cognitive impairment by exosome transfer into damaged neurons and astrocytes. Sci Rep 2016;6:24805.

5. Ren G, Chen X, Dong F, et al. Concise review: mesenchymal stem cells and translational medicine: emerging issues. Stem Cells Transl Med 2012;1:51-8.

6. Hao L, Hailun Z, Qi W, et al. [Changes in bone marrow mesenchymal stem cells osteogenesis by the regulation of Lnk/stem cell factor-cKit signaling]. Hua Xi Kou Qiang Yi Xue Za Zhi 2015;33:633-7.

7. Luo F, Liu T, Wang J, et al. Bone marrow mesenchymal stem cells participate in prostate carcinogenesis and promote growth of prostate cancer by cell fusion in vivo. Oncotarget 2016;7:30924-34.

8. Xiao WZ, Gu XC, Hu B, et al. Role of microRNA129-5p in osteoblast differentiation from bone marrow mesenchymal stem cells. Cell Mol Biol (Noisy-le-grand) 2016;62:95-9. 
9. Zheng S, Yang J, Tang Y, et al. Effect of bone marrow mesenchymal stem cells transplantation on the serum and liver HMGB1 expression in rats with acute liver failure. Int J Clin Exp Pathol 2015;8:15985-92.

10. Deb A, Wang S, Skelding KA, et al. Bone marrow-derived cardiomyocytes are present in adult human heart: A study of gender-mismatched bone marrow transplantation patients. Circulation 2003;107:1247-9.

11. Bader AM, Klose K, Bieback K, et al. Hypoxic Preconditioning Increases Survival and Pro-Angiogenic Capacity of Human Cord Blood Mesenchymal Stromal Cells In Vitro. PLoS One 2015;10:e0138477.

12. Bakshi AA, Bavikar JS, Asegaonkar SB, et al. Evaluation of usefulness of serum insulin as sensitive predictor of cardiovascular dysfunction in obese individuals with normal lipid profile. J Clin Diagn Res 2014;8:CC10-2.

13. Barazzoni R, Zanetti M, Gortan Cappellari G, et al. Fatty acids acutely enhance insulin-induced oxidative stress and cause insulin resistance by increasing mitochondrial reactive oxygen species (ROS) generation and nuclear factor-kappaB inhibitor (IkappaB)-nuclear factor-kappaB (NFkappaB) activation in rat muscle, in the absence of mitochondrial dysfunction. Diabetologia 2012;5 5:773-82.

14. Abe $Y$, Watanabe T. Renal tubular dysfunction in patients with molecular defects of the insulin receptor gene. Eur J Pediatr 2014;173:263.

15. Kotobuki N, Hirose M, Takakura Y, et al. Cultured autologous human cells for hard tissue regeneration: preparation and characterization of mesenchymal stem cells from bone marrow. Artif Organs 2004;28:33-9.

16. Hou L, Dong Q, Wu YJ, et al. Gonadotropins facilitate potential differentiation of human bone marrow mesenchymal stem cells into Leydig cells in vitro. Kaohsiung J Med Sci 2016;32:1-9.

17. Ock SA, Baregundi Subbarao R, Lee YM, et al. Comparison of Immunomodulation Properties of Porcine Mesenchymal Stromal/Stem Cells Derived from the Bone Marrow, Adipose Tissue, and Dermal Skin Tissue. Stem Cells Int 2016;2016:9581350.

18. Wang H, Jing C, Tan X, et al. [An experimental study on segmental defects reconstruction of canine mandible with allogenic bone marrow mesenchymal stem cells combined with lyophilized bone]. Zhonghua Kou Qiang Yi Xue Za Zhi 2015;50:720-4.

19. Zhang F, Zhang Z, Sun D, et al. Periostin: A Downstream Mediator of EphB4-Induced Osteogenic Differentiation of Human Bone Marrow-Derived Mesenchymal Stem Cells. Stem Cells Int 2016;2016:7241829.
20. Zhu N, Wang H, Wang B, et al. A Member of the Nuclear Receptor Superfamily, Designated as NR2F2, Supports the Self-Renewal Capacity and Pluripotency of Human Bone Marrow-Derived Mesenchymal Stem Cells. Stem Cells Int 2016;2016:5687589.

21. Al-Hezaimi K, Ramalingam S, Al-Askar M, et al. Realtime-guided bone regeneration around standardized critical size calvarial defects using bone marrow-derived mesenchymal stem cells and collagen membrane with and without using tricalcium phosphate: an in vivo microcomputed tomographic and histologic experiment in rats. Int J Oral Sci 2016;8:7-15.

22. Lee HJ, Oh SH, Jang HW, et al. Long-Term Effects of Bone Marrow-Derived Mesenchymal Stem Cells in Dextran Sulfate Sodium-Induced Murine Chronic Colitis. Gut Liver 2016;10:412-9.

23. Shangguan L, Li X, Wang Z, et al. [Transforming growth factor-beta 1 induces bone marrow-derived mesenchymal stem cells to differentiate into cancer-associated fibroblasts]. Zhonghua Zhong Liu Za Zhi 2015;37:804-9.

24. Sun Q, Yang Y, Li X, et al. Folate deprivation modulates the expression of autophagy- and circadian-related genes in HT-22 hippocampal neuroncells through GR-mediated pathway. Steroids 2016;112:12-9.

25. Walter C, Clemens LE, Muller AJ, et al. Activation of AMPK-induced autophagy ameliorates Huntington disease pathology in vitro. Neuropharmacology 2016;108:24-38.

26. Li F, Zheng X, Liu Y, et al. Different Roles of CHOP and JNK in Mediating Radiation-Induced Autophagy and Apoptosis in Breast Cancer Cells. Radiat Res 2016;185:539-48.

27. Sano O, Kazetani K, Funata M, et al. Vacuolin-1 inhibits autophagy by impairing lysosomal maturation via PIKfyve inhibition. FEBS Lett 2016;590:1576-85.

28. Chen K, Li J, Li S, et al. 15d-PGJ2 alleviates ConAinduced acute liver injury in mice by up-regulating HO-1 and reducing hepatic cell autophagy. Biomed Pharmacother 2016;80:183-92.

29. Kesidou E, Lagoudaki R, Touloumi O, et al. Autophagy and neurodegenerative disorders. Neural Regen Res 2013;8:2275-83.

30. Liu B, Cao Y, Jiang H, et al. Autophagy facilitates the sorafenib resistance of hepatocellular carcinoma cells. West Indian Med J 2013;62:698-700.

31. Huang H, Li X, Zhuang Y, et al. Class A scavenger receptor activation inhibits endoplasmic reticulum stress-induced autophagy in macrophage. J Biomed Res 2014;28:213-21. 
32. Ahmed K, Jeong MH, Chakraborty R, et al. Coronary Stents in Patients with ST-Elevation Myocardial Infarction and Chronic Kidney Disease Undergoing Primary Percutaneous Coronary Intervention. Korean Circ J 2012;42:830-8.

33. Di Michele S, Mirabelli F, Galzerano D, et al. An unusual myocardial infarction. Echo Res Pract 2014;1:K9-K12.

34. Kalantari Meibodi M. Door to Electrocardiography (ECG) and Needle Times in Patients with Myocardial Infarction. Emerg (Tehran) 2014;2:150.

35. Bashar T, Akhter N. Study on oxidative stress and antioxidant level in patients of acute myocardial infarction before and after regular treatment. Bangladesh Med Res Counc Bull 2014;40:79-84.

36. Nagaya N, Kangawa K, Itoh T, et al. Transplantation of mesenchymal stem cells improves cardiac function in a rat model of dilated cardiomyopathy. Circulation 2005;112:1128-35.

37. Cruz FF, Rocco PR. Hypoxic preconditioning enhances mesenchymal stromal cell lung repair capacity. Stem Cell Res Ther 2015;6:130.

38. Qin HH, Filippi C, Sun S, et al. Hypoxic preconditioning potentiates the trophic effects of mesenchymal stem cells on co-cultured human primary hepatocytes. Stem Cell Res Ther 2015;6:237.

39. Rosová I, Dao M, Capoccia B, et al. Hypoxic preconditioning results in increased motility and improved therapeutic potential of human mesenchymal stem cells. Stem Cells 2008;26:2173-82.

40. Volkmer E, Kallukalam BC, Maertz J, et al. Hypoxic preconditioning of human mesenchymal stem cells overcomes hypoxia-induced inhibition of osteogenic differentiation. Tissue Eng Part A 2010;16:153-64.

41. Kaarniranta K, Kauppinen A, Blasiak J, et al. Autophagy regulating kinases as potential therapeutic targets for age-related macular degeneration. Future Med Chem 2012;4:2153-61.

42. Li L, Zhang Q, Tan J, et al. Autophagy and hippocampal neuronal injury. Sleep Breath 2014;18:243-9.

43. Zhang $Z$, Yang $M$, Wang $Y$, et al. Autophagy regulates the apoptosis of bone marrow-derived mesenchymal stem cells under hypoxic condition via AMP-activated protein kinase/mammalian target of rapamycin pathway. Cell Biol Int 2016;40:671-85.

44. Liu J, Hao H, Huang H, et al. Hypoxia regulates the therapeutic potential of mesenchymal stem cells through enhanced autophagy. Int J Low Extrem Wounds 2015;14:63-72.
45. Mo Y, Tang L, Ma Y, et al. Pramipexole pretreatment attenuates myocardial ischemia/reperfusion injury through upregulation of autophagy. Biochem Biophys Res Commun 2016;473:1119-24.

46. Bandara N, Gurusinghe S, Chen H, et al. Minicircle DNA-mediated endothelial nitric oxide synthase gene transfer enhances angiogenic responses of bone marrowderived mesenchymal stem cells. Stem Cell Res Ther 2016;7:48.

47. Barlow AD, Thomas DC. Autophagy in diabetes: beta-cell dysfunction, insulin resistance, and complications. DNA Cell Biol 2015;34:252-60.

48. Barlow J, Jensen VH, Jastroch M, et al. Palmitate-induced impairment of glucose-stimulated insulin secretion precedes mitochondrial dysfunction in mouse pancreatic islets. Biochem J 2016;473:487-96.

49. Bertolo A, Mehr M, Janner-Jametti T, et al. An in vitro expansion score for tissue-engineering applications with human bone marrow-derived mesenchymal stem cells. J Tissue Eng Regen Med 2016;10:149-61.

50. Ferris DJ, Frisbie DD, Kisiday JD, et al. Clinical outcome after intra-articular administration of bone marrow derived mesenchymal stem cells in 33 horses with stifle injury. Vet Surg 2014;43:255-65.

51. Wong KL, Lee KB, Tai BC, et al. Injectable cultured bone marrow-derived mesenchymal stem cells in varus knees with cartilage defects undergoing high tibial osteotomy: a prospective, randomized controlled clinical trial with 2 years' follow-up. Arthroscopy 2013;29:2020-8.

52. Sohn HS, Heo JS, Kim HS, et al. Duration of in vitro storage affects the key stem cell features of human bone marrow-derived mesenchymal stromal cells for clinical transplantation. Cytotherapy 2013;15:460-6.

53. Clé DV, Santana-Lemos B, Tellechea MF, et al. Intravenous infusion of allogeneic mesenchymal stromal cells in refractory or relapsed aplastic anemia. Cytotherapy 2015;17:1696-705.

54. Jiang S, Xia M, Yang J, et al. Novel insights into a treatment for aplastic anemia based on the advanced proliferation of bone marrow-derived mesenchymal stem cells induced by fibroblast growth factor 1 . Mol Med Rep 2015;12:7877-82.

doi: 10.21037/sci.2017.08.01

Cite this article as: $\mathrm{Li} \mathrm{JR,} \mathrm{Qu} \mathrm{TT.} \mathrm{Into} \mathrm{the} \mathrm{eyes} \mathrm{of} \mathrm{bone}$ marrow-derived mesenchymal stem cells therapy for myocardial infarction and other diseases. Stem Cell Investig 2017;4:69. 